\title{
Solitary Langerhans Cell Histiocytosis of Skull: Case Report with One-year Follow-up
}

\author{
Muhammad Junaid ${ }^{1}$, Syed Sarmad Bukhari ${ }^{3}$ and Ali Afzal ${ }^{2}$ \\ ${ }^{1}$ Department of Neurosurgery, PNS Shifa Hospital, Karachi, Pakistan \\ ${ }^{2}$ Department of Neurosurgery, Jinnah Postgraduate Medical Centre, Karachi, Pakistan \\ ${ }^{3}$ Department of Neurosurgery, The Aga Khan University Hospital, Karachi, Pakistan
}

\begin{abstract}
Langerhans cell histiocytosis ( $\mathrm{LCH}$ ) is a rare disorder characterised by increased production of Langerhans-type histiocytes. It is more common in the pediatric age group with a predilection for osseous involvement, though any organ may be involved. A 10year male child was brought to the neurosurgical clinic with a slow growing painful tender mass on the head. Initial attempt to biopsy the lesion failed due to excessive bleeding. It was later imaged and removed with a frontal craniotomy. Histopathological evaluation along with immunohistochemistry revealed the true nature of the lesion. Follow-up revealed complete excision of the lesion and no recurrence at one-year after surgery.
\end{abstract}

Key Words: Langerhans cell histiocytosis, Pediatric age, Skull.

How to cite this article: Junaid M, Bukhari SS, Afzal A. Solitary Langerhans Cell Histiocytosis of Skull: Case Report with One-year Follow-up. J Coll Physicians Surg Pak 2021; 31(08):989-991.

\section{INTRODUCTION}

Langerhans cell histiocytosis ( $\mathrm{LCH})$ is a rare disorder characterised by increased production of Langerhans-type histiocytes. It is more common in the pediatric age group with a predilection for osseous involvement, though any organ may be affected. The estimated annual incidence varies between 1 to 7 cases per million of the population. ${ }^{1}$ Among children less than two years of age, nearly $50 \%$ have disseminated $\mathrm{LCH}$ with organ dysfunction and a more serious grade of the disease that may be fatal. ${ }^{2}$ The prevalence is higheramong Whites; and males are affected more than females, with a male-to-female ratio of $2: 1{ }^{3}$ The skull is the most common site involved, with the frontal and parietal bones being most often involved. The disease can be localised or systemic with a wide array of clinical presentations. Children with skull lesions usually present with a tender enlarging mass that may be associated with an antecedent history of head trauma. ${ }^{4-6} \mathrm{We}$, herein, report a case of a 10 -year male child who presented with a scalp swelling that was surgically removed. Histopathological evaluation along with immunohistochemistry revealed the true nature of the lesion. Follow-up revealed complete excision of the lesion and no recurrence atone-year aftersurgery.

Correspondence to: Dr. Syed Sarmad Bukhari, Department of Neurosurgery, The Aga Khan University Hospital, Karachi, Pakistan

E-mail: sarmadbukhari@gmail.com

Received: April 22, 2020; Revised: August 30, 2020;

Accepted: September 28, 2020

DOI: https://doi.org/10.29271/jcpsp.2021.08.989

\section{CASE REPORT}

A 10-year - male child was brought to the neurosurgical clinic with a slow growing painful tender mass on the right side of his forehead. On a prior occasion, an incisional biopsy was attempted at another centre, which was abandoned due to profuse bleeding. On clinical examination, the swelling was firm and appeared to be in continuation with the surface of the skull. Overlying skin was free from the lesion and was unremarkable in appearance. He had no other symptoms and neurological examination wasunremarkable.

His magnetic resonance imaging (MRI) brain with contrast was done to rule out intracranial extension. It showed an intensely homogenous contrast enhancing space occupying lesion in the right frontal bone with involvement of the underlying dura (Figure $1 \mathrm{~A}$ and $1 \mathrm{~B}$ ). The dura showed abnormal thickening and contrast enhancement. There was also mass effect over the underlying brain. No other lesions were found.

The patient was planned for a craniotomy under general anaesthesia. He underwent a right frontal craniotomy and excision of the lesion with the involved dura being removed as well. The lesion was highly vascular, but successfully removed and the skull defect was covered with a titanium mesh for cosmesis (Figure 2).

Postoperatively, he had an uneventful recovery. Postoperative MRI showed complete resection of the lesion (Figure 3). Histopathologic evaluation along with immunohistochemistry showed features consistent with LCH (Figure 4). No recurrence was noted at one-year follow-up. 


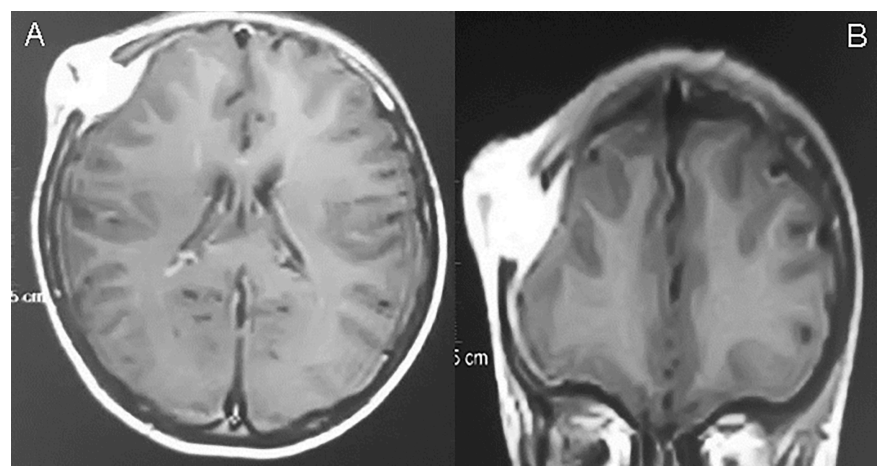

Figure 1 (A, B): Preoperative MRI brain with contrast demonstrates the right frontal lesion involving the bone and underlying dura with intense post-contrastenhancement.

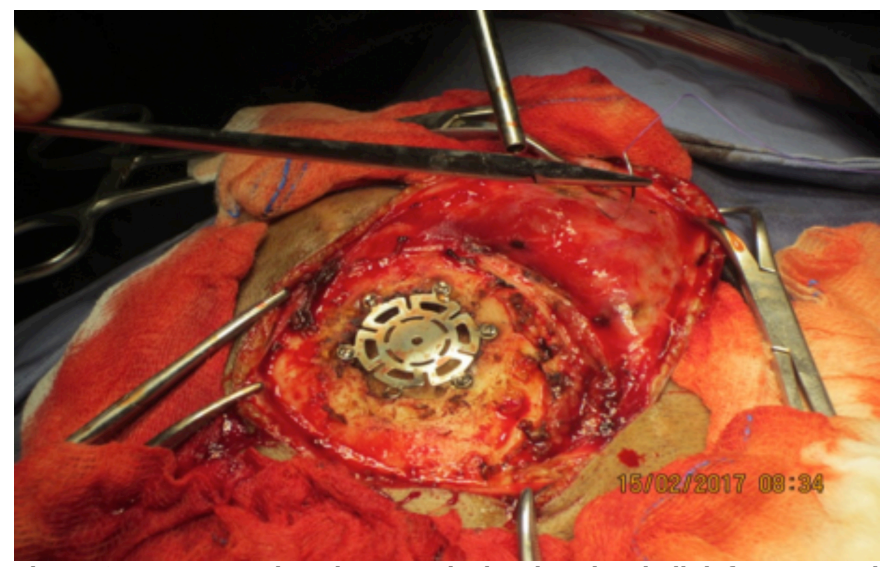

Figure 2: Intraoperative photograph showing the skull defect covered with a titanium mesh.

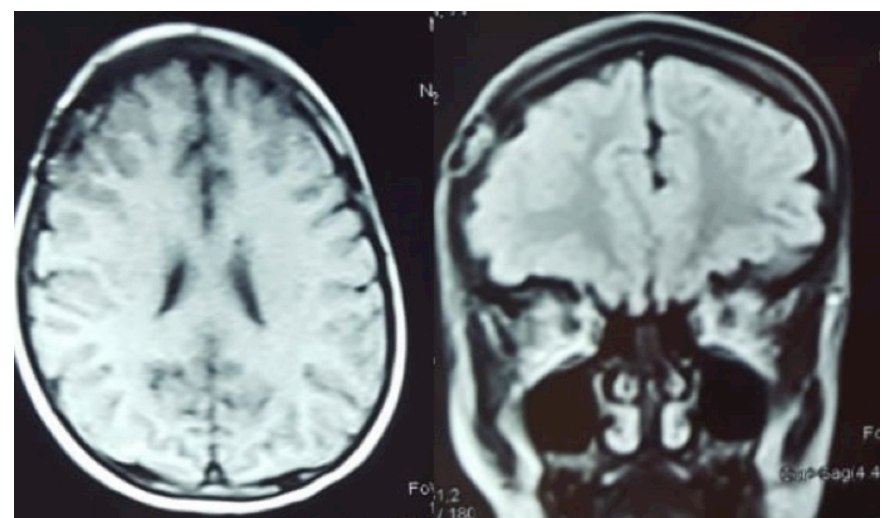

Figure 3: Postoperative MRI scan showing complete excision of the lesion.

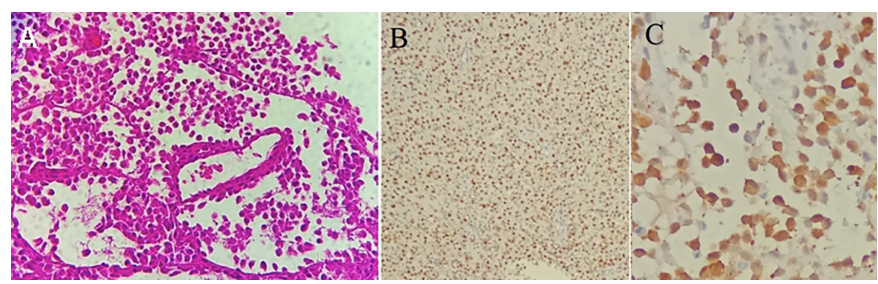

Figure 4: (A) Micrograph demonstrating histiocytes with some atypical cells and a large population of eosinophils. (B) Micrograph showing immunostaining for CD1 alpha and (C) S-100.

\section{DISCUSSION}

LCH is a clonal disorder regarded as localised proliferation of Langerhans cells in connective tissues like bone. It is also known as histiocytosis $X$ or eosinophilic granuloma. The exact aetiology and pathogenesis remain unclear. Bone lesions are the most common manifestations of $\mathrm{LCH}$; they occur in $80 \%-95 \%$ of children with LCH. A predilection for hematopoietically active medullary sites exists; the skull is most frequently involved. ${ }^{4,5}$

The skull lesions usually involve the orbit and the cranial base producing the classic triad of bony defects, exophthalmos and diabetes insipidus. ${ }^{4}$ The possible complications and sequelae of $\mathrm{LCH}$ are site-dependant along with the extent of the lesion. CNS involvement in LCH manifests as altered neurologic function and most children with bony involvement present with symptoms related to bone pain or a localised mass. The treatment of LCH depends on the extent of the disease. Surgical curettage remains the best treatment modality for single osseous lesions. ${ }^{6}$ In localised disease, the prognosis remains excellent.

Both radiology and histology are needed for establishing the diagnosis of $\mathrm{LCH}$. ${ }^{7} \mathrm{LCH}$ is characterised by the presence of proliferative Langerhans cells and eosinophils. ${ }^{4}$ Positive staining for S-100 protein, CD1a and anti-langerin (CD207) through immunohistochemistry is very helpful in establishing the diagnosis. ${ }^{1,2,4}$ On plain skull X-rays, it is seen as a sharp punched-out rounded osteolytic lesion with well demarcated borders. Addition of 3D CT increases the diagnostic accuracy, particularly in small osteolytic lesions. ${ }^{1}$ The outer table is more affected than the inner table or diploe, so lesions may show "beveled" edges resembling a shallow " $\mathrm{V}$ "-shaped groove in CT scans, ${ }^{5,8}$ that can also detect the extent of bony destruction and soft tissue involvement. ${ }^{9} \mathrm{MRI}$ aids in delineating the true extent of soft tissue lesions and their relations to adjacent brain parenchyma and dura mater. ${ }^{4}$

Our patient presented initially with an enlarging painful tender mass. Rawlings and Wilkins presented one of the largest case series of patients with solitary eosinophilic granuloma of the skull; and they reported that all their 26 patients presented with a painful, or at the very least, tender-to-touch lesions. ${ }^{6}$ On the other hand, painless and nontender skull LCH lesions have also been reported and appear to be not uncommon in the literature. ${ }^{7,10}$ Most pediatric patients with a skull LCH lesion will present with an enlarging scalp mass. ${ }^{4,6,8}$ An association between the diagnosis of $\mathrm{LCH}$ and preceding trauma has been observed and reported. ${ }^{1,3,6}$ In the series of Rawlings and Wilkins, they reported that $38 \%$ of their cases were preceded by trauma. ${ }^{7}$ Others have reported antecedant head trauma in $33-50 \%$ of their reported cases, ${ }^{4}$ but the pathophysiology behind the occurrence of LCH after trauma is still not clear. ${ }^{3}$ Some authors believe that trauma is just an event that attracts the attention to previously unnoticed LCH lesions. ${ }^{3,4}$

In conclusion, LCH should be considered in the differential diagnosis of enlarging painful mass lesions of the skull in the pediatric age group. Imaging and histopathology provide the definitive diagnosis. Surgery is often curative in focal and solitary lesions of the skull. 


\section{PATIENT'S CONSENT:}

Informed consent was obtained from patient's guardian to publish the data concerning this case.

\section{CONFLICT OF INTEREST:}

The authors declared no conflict of interest.

\section{AUTHORS' CONTRIBUTION:}

MJ: Data acquisition and analysis, interpretation, critical revision, final approval.

AA: Conception and design, interpretation, critical revision, finalapproval.

SSB: Data acquisition and analysis, interpretation, critical revision, final approval

\section{REFERENCES}

1. Bakhaidar MG, Alghamdi FA, Baeesa SS. Spontaneous extradural hemorrhage due to Langerhans cell histiocytosis of the skull in a child: A rare presentation. J Pediatr Neurosci 2016; 11(1): 52-55. doi: 10.4103/1817- 1745. 181248.

2. Mosiewicz A, Rola R, Jarosz B, Trojanowska A, Trojanowski $T$. Langerhans cell histiocytosis of the parietal bone with epidural and extracranial expansion - case report and a review of the literature. Neurol Neurochir Pol 2010; 44(2):196-203. doi: 10.1016/s0028-3843(14)60011-6.

3. Lee YS, Kwon JT, Park YS. Eosinophilic granuloma presenting as an epidural hematoma and cyst. J Korean
Neurosurg Soc 2008; 43(6):304-6. doi: 10.3340/jkns. 2008.43.6.304.

4. Azouz EM, Saigal G, Rodriguez MM, Podda A. Langerhans' cell histiocytosis: Pathology, imaging and treatment of skeletal involvement. Pediatr Radiol 2005; 35(2):103-15. doi: 10.1007/s00247-004-1262-0.

5. Lee KW, McLeary MS, Zuppan CW, Won DJ. Langerhans' cell histiocytosis presenting with an intracranial epidural hematoma. Pediatr Radiol 2000; 30(5):326-8. doi: 10.1007/s002470050750.

6. Chen HC, Shen WC, Chou DY, Chiang IP. Langerhans cell histiocytosis of the skull complicated with an epidural hematoma. AJNR Am J Neuroradiol 2002; 23(3):493-5. doi.org/10.1007/ s0 02470050750.

7. Rawlings CE, 3rd, Wilkins RH. Solitary eosinophilic granuloma of the skull. Neurosurgery 1984; 15(2):155-61. doi: 10.1227/00006123-198408000-00001.

8. Pawar RV, Hagiwara M, Milla S, Wisoff J, George AE. Eosinophilic granuloma presenting as post-traumatic scalp hematoma with epidural hemorrhage. A case report. Neuroradiol J 2011; 24(5):767-1. doi: 10.1177/ 197140091102400516.

9. Cho DY, Liau WR, Chiang IP. Eosinophilic granuloma with acute epidural hematoma: A case report. Pediatr Neurosurg 2001; 35(5):266-9. doi: 10.1159/000050434.

10. Manaka S, Izawa M, Nawata H. Skull tumor simulating sinus pericranii. Case report. J Neurosurg 1977; 46(5):671-3. doi: 10.3171/jns.1977.46.5.0671. 